\title{
Polysomy and p16 deletion by fluorescence in situ hybridization in the diagnosis of indeterminate biliary strictures
}

Tamas A. Gonda, MD, ${ }^{1}$ Michael P. Glick, MD ${ }^{1}$ Amrita Sethi, MD, ${ }^{1}$ John M. Poneros, MD, ${ }^{1}$ Walter Palmas, $M D,{ }^{3}$ Shahzad Iqbal, MD, ${ }^{1}$ Susana Gonzalez, MD, ${ }^{1}$ Subhadra V. Nandula, MD, ${ }^{2}$ Jean C. Emond, MD, ${ }^{4}$ Robert S. Brown, MD, MPH, ${ }^{1,4}$ Vundavalli V. Murty, PhD, ${ }^{2}$ Peter D. Stevens, MD $^{1}$

New York, New York, USA

Background: The diagnosis of indeterminate biliary strictures is limited because of the low sensitivity of cytology. However, an accurate diagnosis of malignancy is critical in the management of patients with suspected biliary malignancy. Testing for chromosomal aneuploidy by fluorescence in situ hybridization (FISH) may increase the yield.

Objective: To evaluate the diagnostic accuracy of FISH in indeterminate biliary strictures and the additional value of including deletion of 9p21 (p16) in the diagnostic criteria of malignant biliary strictures.

Design: Retrospective review.

Setting: Academic medical center.

Patients: This study involved 76 consecutive patients who were seen for the evaluation of indeterminate strictures at our institution. These patients were screened, and 50 patients with either a final pathologic diagnosis or $\geq 12$ months' conclusive follow-up were included in the analysis.

Main Outcome Measurements: Sensitivity, specificity, and area under the curve (AUC) analysis of cytology alone compared with the presence of FISH polysomy versus FISH polysomy and 9p21 deletion.

Results: The presence of increased copy numbers (polysomy) of chromosome 3, 7, or 17 by FISH increased the sensitivity of brush cytology from $21 \%$ to $58 \%$, and when the presence of $9 \mathrm{p} 21$ deletion was included, the sensitivity increased to $89 \%$. The specificity of FISH was $97 \%$ (vs 100\% for cytology). The accuracy of cytology combined with FISH polysomy (AUC $=0.93$ ) or p16 deletion was significantly greater than the accuracy of cytology alone (AUC $0.6 ; P<.001$ ) or even cytology combined with FISH polysomy (AUC $=0.77 ; P \leq .05$ ).

Limitations: Sample size. There is a relatively high incidence of malignant biliary strictures in the entire cohort but low incidence among primary sclerosing cholangitis patients, and the majority of cancers are cholangiocarcinomas (as opposed to pancreatic).

Conclusion: FISH significantly improves the diagnostic accuracy of brush cytology in indeterminate biliary strictures. In our series, the addition of 9p21 deletion to FISH polysomy and cytology further improved sensitivity. This suggests that 9p21 deletion may be added to the diagnostic criteria in indeterminate strictures. (Gastrointest Endosc 2012;75:74-9.)

Abbreviations: AUC, area under the curve; CEP, chromosome enumeration probe; CI, confidence interval; FISH, fluorescence in situ bybridization; PSC, primary sclerosing cholangitis; ROC, receiver operating characteristic.

DISCLOSURE: All authors disclosed no financial relationships relevant to this publication.

Copyright $\odot 2012$ by the American Society for Gastrointestinal Endoscopy 0016-5107/\$36.00

doi:10.1016/j.gie.2011.08.022

Received April 1, 2011. Accepted August 14, 2011.
Current affiliations: Division of Digestive and Liver Diseases (1), Department of Pathology and Cell Biology (2), Department of General Medicine (3), Department of Surgery (4), Columbia University Medical Center, New York, New York, USA.

Reprint requests: Tamas A. Gonda, MD, Division of Digestive and Liver Diseases, Columbia University Medical Center, $161 \mathrm{Ft}$. Washington Avenue, Suite 861, New York, NY 10025.

If you would like to chat with an author of this article, you may contact $\mathrm{Dr}$ Gonda at tgonda@gmail.com. 
Biliary strictures are most often evaluated by ERCP or percutaneous transhepatic cholangiography. Although significant advances have been made in the available therapies of both benign and malignant strictures, diagnostic distinction between these two entities remains one of the greatest challenges in the field., ${ }^{1,2} \mathrm{~A}$ biliary stricture is designated as indeterminate when imaging and other modalities fail to reveal an associated mass or evidence of metastatic disease or when there is no associated etiology, such as stone disease or recent bile duct trauma.

It is suspected that the growth pattern and anatomic location makes sampling, and therefore accurate diagnosis, especially difficult. ${ }^{3}$ Advances in chemoradiation therapy and intraductal therapies (ie, photodynamic therapy) as well as liver transplantation protocols have yielded dramatic survival benefits $s^{4,5}$ in cholangiocarcinoma, and this further emphasizes the need for accurate and timely diagnosis.

Multiple procedural modalities and sampling techniques have been used for the evaluation of indeterminate strictures. Cytology, obtained by brushing of strictures during either ERCP or percutaneous transhepatic cholangiography, has been the mainstay of diagnosis. Given that the specificity of cytology is almost $100 \%$, there is little need for further evaluation with a positive result. However, brush cytology has poor diagnostic sensitivity that ranges from $5 \%$ to $40 \%{ }^{6,7}$ Direct bile-duct biopsies, cholangioscopically guided biopsies, and improved imaging of the biliary epithelium may further enhance accuracy, but these modalities require additional procedures and expertise. The sensitivity of these modalities is also variable.

Fluorescence in situ hybridization (FISH) assesses the presence of chromosomal aneuploidy (abnormality in chromosome number) and uses fluorescence-labeled probes to evaluate increases or decreases in chromosome number. ${ }^{8}$ This analysis is performed on brush specimens and can provide accurate diagnosis when only a few neoplastic cells are present in the sample. Previous studies performed predominantly by researchers at the Mayo Clinic have evaluated several chromosomal regions and have determined that polysomy of chromosomes 3, 7, and 17 centromeric regions was most useful for the diagnosis of malignancy. These studies have demonstrated that FISH polysomy improved sensitivity (45\%$59 \%$ ) when combined with cytology versus cytology alone, whereas specificity remained near 100\%.7,9,10 When trisomy was considered positive, sensitivity improved, but specificity decreased, especially in patients with primary sclerosing cholangitis (PSC) (the specificity was $93 \%-95 \%$ in patients without PSC vs $70 \%-87 \%$ in patients with PSC). These series did not include p16 deletion among the positive diagnostic criteria for FISH.

The tumor suppressor gene $\mathrm{p} 16^{\mathrm{INK} 4 \mathrm{a}}$ regulates cell cycle entry and is frequently inactivated by genetic (mutations or deletions) and epigenetic (promoter hypermethylation)

\section{Take-home Message}

- Complementing prior works on the use of fluorescence in situ hybridization in biliary diagnostics, the results of this study suggest that the inclusion of polysomy or $\mathrm{p} 16$ deletion improved accuracy of cancer diagnosis.

mechanisms. Although the frequent decrease in p16 expression observed in cholangiocarcinoma may be due to any of these mechanisms, prior works have suggested that there is allelic loss of 9p21 in the majority of cases. We hypothesized, based on this observation, that the detection of homozygous or heterozygous deletion of the 9p21 locus (p16) can increase the diagnostic yield in biliary malignancy. ${ }^{11-13}$ Therefore, the goal of our study was to validate the diagnostic accuracy of FISH polysomy and determine whether inclusion of p16 deletion in the diagnostic criteria for malignancy significantly increases the sensitivity.

\section{METHODS}

\section{Patient selection}

This study was approved by the Institutional Review Board of Columbia University. All consecutive patients who underwent ERCP or percutaneous transhepatic cholangiography at our institution for the evaluation of indeterminate strictures and had biliary brush cytology and FISH specimens obtained between February 2008 and February 2010 were included. Patients were excluded if they had biliary sampling without a dominant stricture (such as in PSC) or if sampling was done for abnormalities other than a stricture on the cholangiogram. An indeterminate stricture was defined as a biliary duct narrowing or obstruction that was not associated with a known malignancy or mass and was not associated with recent bile duct trauma, surgery, or stone disease. Only patients who had $\geq 12$ months' follow-up or a final pathologic diagnosis were included in the study. A stricture was designated as benign if at $\geq 12$ months' follow-up repeat imaging, repeat procedure, or repeat laboratory testing was performed, and these documented the resolution of the stricture or prior abnormalities. A diagnosis of malignant stricture was made when either subsequent biopsy or cytology specimens demonstrated malignancy, final pathology was obtained by surgery, or if the cause of death was biliary malignancy. The diagnosis of PSC was made based on cholangiographic findings associated with laboratory abnormalities or liver biopsy and lack of other etiology as the cause of stricture. Cholangitis was diagnosed when biliary obstruction was accompanied by fever, shock, mental status changes, or an elevated white blood cell count. 


\section{Specimen collection}

Samples were obtained by using two standard cytology brushes (Boston Scientific, Cambridge, Mass). The first brush was always used to obtain cytology specimens, which were then analyzed by a pathologist and classified as "no malignancy," "atypical," "suspicious," or "malignant." A positive result for the purposes of this study included only malignant specimens (among the few cytology-positive cases, there were no suspicious diagnoses). For the FISH analysis, a separate brush was used (always following the taking of the cytology specimen). Specimens were obtained with at least 10 to-and-fro motions at the site of the dominant stricture for both cytology and FISH specimens. The brush was cut and placed into 15 $\mathrm{mL}$ of ThinPrep CytoLyt solution (Marlborough, Mass) and was transferred to the cytogenetics laboratory within 24 hours.

\section{Fluorescence in situ hybridization}

Cells were fixed twice by using freshly made 3:1 methanol-acetic acid. Cytospin cell preparations were made and stored at room temperature. FISH was performed by using the standard protocols and commercially available probes specific to centromeres of chromosomes 3 (chromosome enumeration probe [CEP] 3, orange), 7 (CEP 7, green), and 17 (CEP 17, aqua) in 1 hybridization and p16 (9p21, orange/centromere 9, green) in the second hybridization. An additional hybridization probe (Urovysion; Abbott Molecular, Des Plaines, Ill) consisting of CEP 3 (orange), CEP 7 (green), CEP 17 (aqua), and 9p21 (p16, gold) was used in some cases. Fluorescent signals were scored on 25 to 100 cells on 4',6-diamidino-2-pheylindole (DAPI)-stained slides by using the Cytovision Imaging system attached to a Nikon Eclipse 600 microscope (Applied Imaging, Santa Clara, Calif). The following criteria were applied to select the cells to score: larger nuclear size, irregular nuclear shape, patchy DAPI stain, and clustered but not overlapped cells. Signals were recorded as polysomy when there was a gain of two or more chromosomes in at least 5 cells. Homozygous deletion of 9p21/ p16 was considered when both copies were absent in at least 10 cells as per scoring criteria suggested by the manufacturer (Abbott Molecular, Des Plaines, Ill), or if there was heterozygous deletion in at least $6 \%$ of the total cells analyzed, the variation that was established by using normal specimens in the laboratory. We established normal cutoff values in the laboratory for each of the probe set combinations by using normal tissues and from normal biliary tissue obtained in prior resections of benign disease. For the CEP 3, CEP 7, and CEP 17 probe combinations, the normal variation for at least two trisomies was $2 \%$ at a $95 \%$ confidence interval (CI). For the 9p21/CEP 9 probe, it was $0 \%$ for homozygous deletion and $6 \%$ for heterozygous deletion at a 95\% CI. Examples of these chromosomal abnormalities are shown in Figure 1.
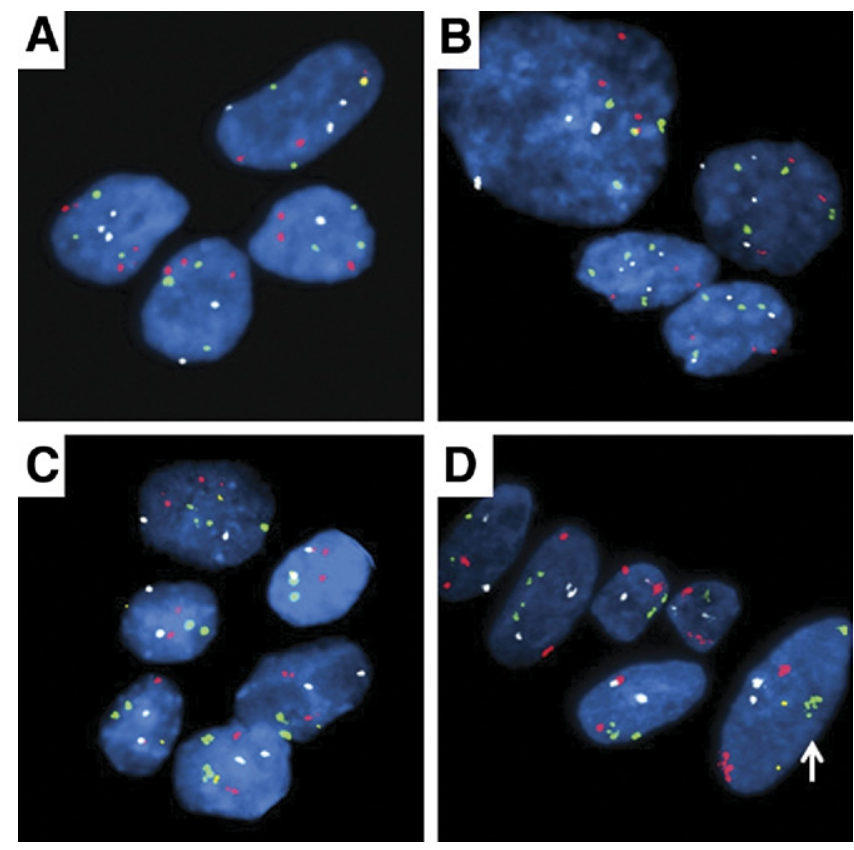

Figure 1. Examples of cytogenetic abnormalities seen in cholangiocarcinoma. A, Trisomy of CEP 3, 7, and 17 demonstrated by 3 copies of red, green, and aqua color probes in 3 cells. B, Polysomy ( 4 copies/cell) of CEP 3, 7, and 17. C, Heterozygous deletion of 9p21 demonstrated by the presence of only 1 gold-colored probe. The cells contain two copies of CEP 3, 7, and 17. D, Homozygous deletion of 9p21 demonstrated by the absence of gold-colored probes in 5 abnormal cells compared with 1 normal cell (arrow) that contains two copies. All cells contain two copies of CEP 3, 7, and 17

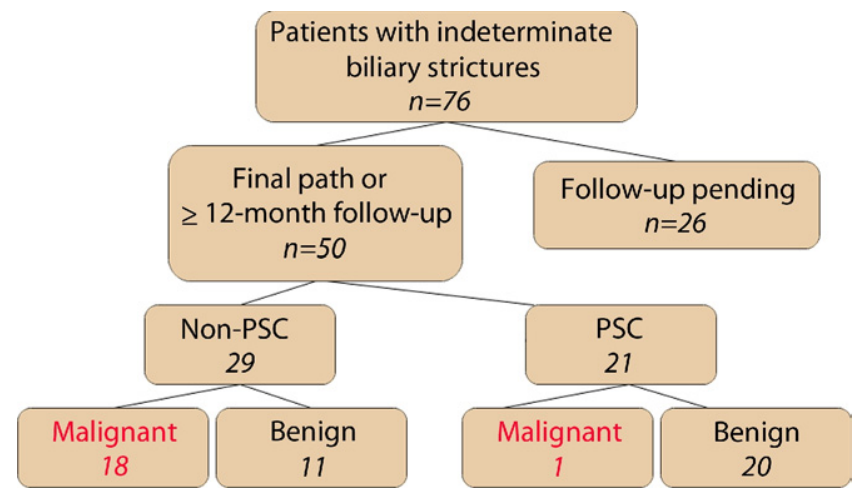

Figure 2. Study design. Seventy-six patients were screened, and 50 were included in the analysis. PSC, primary sclerosing cholangitis.

\section{Statistical analysis}

Binomial exact 95\% CIs were estimated for all sensitivities and specificities. Receiver operating characteristic (ROC) curves were built by using MedCalc 11.3 (MedCalc software; Mariakerke, Belgium). The binomial exact method was applied to estimate the $95 \%$ CI of the areas under the curve (AUC), and pair-wise comparisons of ROC curves were performed by using a nonparametric approach. ${ }^{14}$ Although our study was retrospective, we estimated power for a paired ROC comparison in $n=50$, between FISH polysomy or cytology and FISH polysomy 


\begin{tabular}{lccccc} 
Characteristic* & Cytology & FISH polysomy & $\begin{array}{c}\text { FISH polysomy or } \\
\text { cytology }\end{array}$ & $\begin{array}{c}\text { FISH polysomy or } \\
\text { 9p21 deletion }\end{array}$ & $\begin{array}{c}\text { FISH polysomy, } \\
\text { 9p21 deletion, or } \\
\text { cytology }\end{array}$ \\
\hline Sensitivity & $4 / 19(21)$ & $9 / 19(47)$ & $11 / 19(58)$ & $16 / 19(84)$ & $17 / 19(89)$ \\
Specificity & $31 / 31(100)$ & $30 / 31(97)$ & $30 / 31(97)$ & $30 / 31(97)$ & $30 / 31(97)$ \\
\hline PPV & $4 / 4(100)$ & $9 / 10(90)$ & $11 / 12(92)$ & $16 / 17(94)$ & $17 / 18(94)$ \\
\hline NPV & $31 / 46(67)$ & $30 / 40(75)$ & $30 / 38(79)$ & $30 / 33(91)$ & $30 / 32(94)$ \\
\hline AUC $(95 \% \mathrm{Cl})$ & $0.605(0.457-0.741)$ & $0.721(0.576-0.838) \dagger$ & $0.773(0633-0.880) \dagger$ & $0.905(0.788-0.970) \neq$ & $0.931(0.823-0.983) \neq, \Omega$ \\
\hline
\end{tabular}

FISH, Fluorescence in situ hybridization; PPV, positive predictive value; NPV, negative predictive value; $A U C$, area under the curve; $C l$, confidence interval.

*The sensitivity, specificity, NPV, and PPV of different diagnostic strategies when used alone or in combination are shown. Exact Cls were estimated for each AUC by using a binomial distribution.

$\dagger P \leq .05$.

$\ddagger P \leq .001$ represents difference compared with the cytology alone diagnostic strategy (first category).

$\mathbb{S} \leq .05$ represents comparison to the cytology or FISH polysomy (third category).

or cytology combined with 9p21 deletion. For a prespecified alpha $=0.05$, assuming $\mathrm{AUC}=0.70$ for the first strategy based on prior results and a correlation $r=0.6$ between curves, the paired ROC comparison had a power of $60 \%$ to detect an improvement to $\mathrm{AUC}=0.85$ and a power of $86 \%$ for an improvement to AUC $=0.90$ after the addition of 9 p21 deletion.

\section{RESULTS}

\section{Clinical and histologic characteristics}

Seventy-six patients underwent ERCP or percutaneous transhepatic cholangiography, with tissue obtained for cytology, and FISH during the period of the study. A total of 50 patients underwent surgery, reached a final diagnosis, or had reached $\geq 12$ months' of follow-up (Fig. 2). Twentysix patients were not included in the analysis because follow-up had not reached 12 months (15/26) or patients were lost to follow-up (8/26) or died within 12 months of the procedure (3/26). Of the 50 patients, 21 had PSC, and in this group one cholangiocarcinoma was identified. Of the 29 patients without PSC, 19 were found to have malignancy, with 15 cholangiocarcinomas and 3 pancreatic adenocarcinomas diagnosed. The final diagnosis of malignancy was made at the time of resection in 7 cases, by surgical biopsy in 4 cases, by subsequent endoscopic sampling in 5 cases (by intraductal biopsies in 3 and EUS-guided FNA in 2), or based on the stated cause of death in 3 cases. There was an even distribution of proximal (27/50) and distal (23/50) biliary strictures. Nineteen of these patients underwent a prior procedure (ERCP), and 15 patients had a stent placed. None of these patients had a diagnostic cytology, and in all cases a cytology was obtained at the initial procedure. Eight of 15 patients who had stents placed had cancer, and in none of these cases was a cytologic diagnosis made during the second proce- dure. Significantly more men (14) than women (5) were diagnosed with cancer $(P<.05)$.

\section{Diagnostic techniques: combination of polysomy and p16 deletion significantly improves diagnostic sensitivity}

Cytology alone had a sensitivity and specificity of $22 \%$ and $100 \%$, respectively, for diagnosing malignancy. FISH polysomy of chromosome 3,7 , or 17 had a sensitivity and specificity of $47 \%$ and $97 \%$, respectively. When we included 9p21 heterozygous and homozygous deletions, FISH sensitivity increased to $84 \%$, with preserved specificity of $97 \%$. In the majority of these cases, there was either low cellularity $(15 / 17)$ in the specimen, or only benign cells were seen (2/17). Combined cytology and FISH (considered positive if either were positive) yielded a sensitivity of $89 \%$, specificity of $97 \%$, positive predictive value of $94 \%$, and negative predictive value of 94\% (Table 1). Overall, there was one false-positive sample, which had an unusual pattern of chromosomal abnormality, showing tetraploidy of chromosome 9 (9p21) and disomy of chromosomes 3, 7, and 17. It was classified as a positive finding, based on the increase in copy numbers at the 9p21 locus, although the biologic significance of this is not known. This sample was considered positive, based on the presence of an increased copy number and despite not meeting formal criteria for polysomy, and it was included in this group as a false positive. This patient had PSC and subsequently underwent liver transplantation for recurrent cholangitis. No dysplasia was noted in the explanted liver.

Of note, one additional chromosomal abnormality was noted in our series in a patient with PSC who had trisomy of chromosome 7 . However, previous studies have shown this abnormality to be particularly common in PSCassociated nonmalignant strictures, and therefore this finding was not considered positive in this series. ${ }^{15}$ 


\begin{tabular}{|lc|}
\hline $\begin{array}{l}\text { TABLE 2. Frequency of chromosomal abnormalities in } \\
\text { malignant cases }\end{array}$ \\
$\begin{array}{l}\text { Chromosomal abnormality } \\
\text { Aneuploidy chromosome } 3\end{array}$ & No. (\%), $\mathbf{n}=\mathbf{1 9}$ \\
\hline Aneuploidy chromosome 7 & $10(52)$ \\
\hline Aneuploidy chromosome 17 & $11(57)$ \\
\hline Heterozygous 9p21 deletion & $7(36)$ \\
\hline Homozygous 9p21 deletion & $5(26)$ \\
\hline Polysomy without 9p21 deletion & $9(47)$ \\
\hline $9 p 21$ deletion without polysomy & $7(36)$ \\
\hline
\end{tabular}

The overall diagnostic accuracy (or the sensitivity and specificity trade-off) was determined by calculating the AUC for cytology alone versus cytology combined with polysomy versus cytology combined with either polysomy or $9 \mathrm{p} 21$ deletion. Compared with cytology alone, there was a significant difference between FISH polysomy, polysomy or cytology $(P \leq .05)$, polysomy or 9 p21 deletion $(P \leq .001)$, and polysomy or $9 \mathrm{p} 21$ deletion or cytology $(P \leq .05)$. (Table 1$)$.

\section{Pattern of chromosomal abnormalities in biliary malignancy}

In Table 2, we show the diagnostic modality and the pattern of chromosomal abnormalities that were seen in malignant cases. The p16 deletion can be observed frequently in highly aneuploid tumors, but in 5 cases it was the single abnormality noted (in 2 cases only 9p21 homozygous deletion was noted, and in 3 cases only heterozygous deletion was seen). Aneuploidy of chromosome 17 or 9 p21 heterozygous or homozygous deletion, based on the patterns of chromosomal abnormalities noted, would have been sufficient to diagnose all of our cases.

\section{DISCUSSION}

The recent improvement in outcomes of medical and surgical treatment options for malignant biliary strictures highlights the importance of accurate diagnosis of indeterminate strictures. ${ }^{1,4}$ Advances in imaging and cholangioscopy will continue to enhance our ability to characterize these strictures and target samples and perhaps guide therapy. ${ }^{16}$ Inherent to the growth pattern of bile duct cancers, it is likely that both cytology and biopsy will yield only a small amount of malignant cells. FISH allows the detection and recognition of a very small fraction of abnormal cells in a brush specimen and is therefore particularly suited for diagnostic use in these cases.
In our single-center study, FISH clearly increased the diagnostic accuracy of cytology. Our findings validate prior observations $7,10,15,17$ and provide additional data from an independent center as well as new data, with the inclusion of the $9 \mathrm{p} 21$ probe.

Importantly, there was only one false-positive FISH specimen. The significance of an isolated tetraploidy of chromosome 9 (in the absence of an increased copy number of other chromosomal regions) is not known, and further studies may need to assess the independent significance of this abnormality. It is important to note that this abnormality would not meet our formal criteria for malignancy. Prior studies have shown that the majority of falsepositive cytology results also are obtained in patients with PSCs, and certain trisomies are observed in the absence of malignancies. ${ }^{18}$ Further and larger-scale studies will be needed to assess the significance of chromosome 9 tetraploidy.

Although the 9p21 probe has been included in the probe array used by prior studies, this is the first study to report the use of $9 \mathrm{p} 21$ deletion in the absence of other polysomies as part of the diagnostic criteria for malignancy. We propose, based on the significantly improved accuracy resulting from sensitivity increased from $47 \%$ to $84 \%$ and unchanged specificity, that future studies should evaluate and consider the inclusion of 9p21 among the diagnostic criteria of FISH in indeterminate strictures. In this series, two probes (CEP 17 and 9p21) would have been sufficient to diagnose all lesions, and, therefore, further prospective studies may further narrow the number of probes used to diagnose cancer.

Importantly, there was only one cancer that was not diagnosed by FISH and had positive cytology, and there were two cancers with negative FISH and cytology. Two of these patients had distal biliary strictures secondary to pancreatic adenocarcinoma, and one patient had an intrahepatic cholangiocarcinoma. Both of these locations may be difficult to sample with a brush, and this may account for the false-negative results. The incorporation of EUSguided FNA and targeted biliary biopsies will likely improve the diagnostic yield in these anatomic locations.

There are several limitations to this study, which may impact interpretation of the results. Because our sample size was small, there is a chance for a type I error (as manifest in the width of the $95 \%$ CIs of the areas under the ROC curves). There was a relatively high proportion of malignant strictures among the non-PSC cases and, on the contrary, a surprisingly low incidence of aneuploidy as well as malignancy among patients with PSC. The prevalence of cholangiocarcinoma in PSC is between 5\% and $36 \%$, based on prior series, whereas the rate of malignancy in indeterminate strictures is expected to be much higher $(>70 \%) .{ }^{19}$ The likely difference from these numbers may reflect the referral bias of our institution. The spectrum of malignancy, unlike in prior studies, also reflects a higher proportion of cholangiocarcinomas (prior studies included 
a greater number of either pancreatic cancer or metastatic disease). Therefore, it is difficult to ascertain from this study whether FISH has a different role in the case of suspected pancreatic cancer-related indeterminate strictures versus suspected cholangiocarcinomas.

In conclusion, our data and data from the Mayo Clinic support the use of FISH in the diagnostic evaluation of indeterminate biliary strictures. The estimated per-patient cost of a FISH analysis at this institution is $\$ 500$ to $\$ 1000$. Given the economic ramifications of this test, one could consider limiting FISH evaluation to strictures for which cytology was nondiagnostic. Because cytology specimens remain stable for several days in CytoLyt, this timeframe can be sufficient to make a cytologic assessment and proceed to FISH analysis only if a diagnosis is not made. Further prospective studies may be necessary to define the precise diagnostic algorithm of indeterminate stricture evaluation, and these studies will likely answer whether FISH should be included in the primary evaluation of all strictures. Future research also may consider including aneuploidy of other chromosomal regions to increase diagnostic yield.

\section{REFERENCES}

1. Van Beers BE. Diagnosis of cholangiocarcinoma. HPB (Oxford) 2008;10: 87-93.

2. Shaib YH, Davila JA, McGlynn K, et al. Rising incidence of intrahepatic cholangiocarcinoma in the United States: a true increase? J Hepatol 2004;40:472-7.

3. Ponchon T, Gagnon P, Berger F, et al. Value of endobiliary brush cytology and biopsies for the diagnosis of malignant bile duct stenosis: results of a prospective study. Gastrointest Endosc 1995;42:565-72.

4. Rosen CB, Heimbach JK, Gores GJ. Surgery for cholangiocarcinoma: the role of liver transplantation. HPB (Oxford) 2008;10:186-9.

5. Simmons DT, Baron TH, Petersen BT, et al. A novel endoscopic approach to brachytherapy in the management of hilar cholangiocarcinoma. Am J Gastroenterol 2006;101:1792-6.

6. Rösch T, Hofrichter K, Frimberger E, et al. ERCP or EUS for tissue diagnosis of biliary strictures? A prospective comparative study. Gastrointest Endosc 2004;60:390-6.
7. Moreno Luna LE, Kipp B, Halling KC, et al. Advanced cytologic techniques for the detection of malignant pancreatobiliary strictures. Gastroenterology 2006;131:1064-72.

8. Bergquist A, Tribukait B, Glaumann $\mathrm{H}$, et al. Can DNA cytometry be used for evaluation of malignancy and premalignancy in bile duct strictures in primary sclerosing cholangitis? J Hepatol 2000;33:873-7.

9. Kipp BR, Stadheim LM, Halling SA, et al. A comparison of routine cytology and fluorescence in situ hybridization for the detection of malignant bile duct strictures. Am J Gastroenterol 2004;99:1675-81.

10. Fritcher EG, Kipp BR, Halling KC, et al. A multivariable model using advanced cytologic methods for the evaluation of indeterminate pancreatobiliary strictures. Gastroenterology 2009;136:2180-6.

11. Salek $C$, Benesova $L$, Zavoral $M$, et al. Evaluation of clinical relevance of examining K-ras, p16 and p53 mutations along with allelic losses at $9 p$ and $18 q$ in EUS-guided fine needle aspiration samples of patients with chronic pancreatitis and pancreatic cancer. World J Gastroenterol 2007; 13:3714-20.

12. DeHaan RD, Kipp BR, Smyrk TC, et al. An assessment of chromosomal alterations detected by fluorescence in situ hybridization and $\mathrm{p} 16$ expression in sporadic and primary sclerosing cholangitis-associated cholangiocarcinomas. Hum Pathol 2007;38:491-9.

13. Tannapfel A, Benicke M, Katalinic A, et al. Frequency of p16(INK4A) alterations and K-ras mutations in intrahepatic cholangiocarcinoma of the liver. Gut 2000;47:721-7.

14. DeLong ER, DeLong DM, Clarke-Pearson DL. Comparing the areas under two or more correlated receiver operating characteristic curves: a nonparametric approach. Biometrics 1988;44:837-45.

15. Moreno Luna LE, Gores GJ. Advances in the diagnosis of cholangiocarcinoma in patients with primary sclerosing cholangitis. Liver Transpl 2006;12(11 suppl 2):S15-19.

16. Iqbal S, Stevens PD. Cholangiopancreatoscopy for targeted biopsies of the bile and pancreatic ducts. Gastrointest Endosc Clin N Am 2009;19: 567-77.

17. Levy MJ, Baron TH, Clayton AC, et al. Prospective evaluation of advanced molecular markers and imaging techniques in patients with indeterminate bile duct strictures. Am J Gastroenterol 2008;103:1263-73.

18. Ponsioen CY, Lam K, van Milligen de Wit AW, et al. Four years experience with short term stenting in primary sclerosing cholangitis. Am J Gastroenterol 1999;94:2403-7.

19. Barr Fritcher EG, Kipp BR, Slezak JM, et al. Correlating routine cytology, quantitative nuclear morphometry by digital image analysis, and genetic alterations by fluorescence in situ hybridization to assess the sensitivity of cytology for detecting pancreatobiliary tract malignancy. Am J Clin Pathol 2007;128:272-9. 